\title{
SOME APPLICATIONS OF LEGENDRE NUMBERS
}

\author{
PAUl W. HAGGARD \\ Department of Mathematics, East Carolina University \\ Greenville, North Carolina 27858 U.S.A. \\ (Received August 26, 1986)
}

ABSTRACT. The associated Legendre functions are defined using the Legendre numbers. From these the associated Legendre polynomials are obtained and the derivatives of these polynomials at $x=0$ are derived by using properties of the Legendre numbers. These derivatives are then used to expand the associated Legendre polynomials and $x^{n}$ in series of Legendre polynomials. Other applications include evaluating certain integrals, expressing polynomials as linear combinations of Legendre polynomials, and expressing linear combinations of Legendre polynomials as polynomials. A connection between Legendre and Pascal numbers is also given.

KEY WORDS ANV PHRASES. Associated Legendre functions and poxynomials, Legendre polynomials, derivatives of associated Legendre polynomials, serces and integrals of Legendre poxynomials, Legendre and Pascal numbers.

1980 MATHEMATICS SUBJECT CLASSIFICATION CODES. 10A40, $26 \mathrm{C} 99,33 A 45$.

\section{INTRODUCI'ION.}

The Legendre numbers were introduced and many of their elementary properties were developed in [1]. We apply these properties to a variety of problems and the use of Legendre numbers may provide somewhat simpler solutions to the problems.

2. DERIVATIVES OF ASSOCIATED LEGENDRE POLYNOMIALS AT $\mathbf{x}=0$.

For $n$ and $s$ non-negative integers the associated Legendre functions are defined as usual by

$$
\mathrm{P}_{\mathrm{n}}^{\mathrm{s}}(\mathrm{x})=\left(1-\mathrm{x}^{2}\right)^{\mathrm{s} / 2} \mathrm{D}^{s} \mathrm{P}_{\mathrm{n}}(\mathrm{x}),
$$

where $P_{n}(x)$ is a Legendre polynomial and $D^{s} \equiv \frac{d^{s}}{\dot{c} x^{s}}$. Since $P_{n}(x)$ can be expressed, see [1], by using the Legendre numbers, $P_{n}^{i}$, (where $\left.P_{n}^{i}=P_{n}^{i}(x)\right]_{x=0}$ ), as

$$
P_{n}(x)=\sum_{i=0}^{n} \frac{P_{n}^{+} x^{i}}{i !}
$$

Equation (2.1) becomes 


$$
P_{n}^{s}(x)=\left(1-x^{2}\right)^{s / 2} D^{s} \sum_{1=0}^{n} \frac{P_{n}^{i} x^{i}}{1 !}
$$

It is clear that $P_{n}^{s}(x)$ is a polynomial of degree $n$ for $s$ even. Thus, let $s=2 m$ and recall, see [1], that $P_{n}^{i}=0$ for $n$ and $i$ of different parity. Omitting these null terms from (2.3), one has

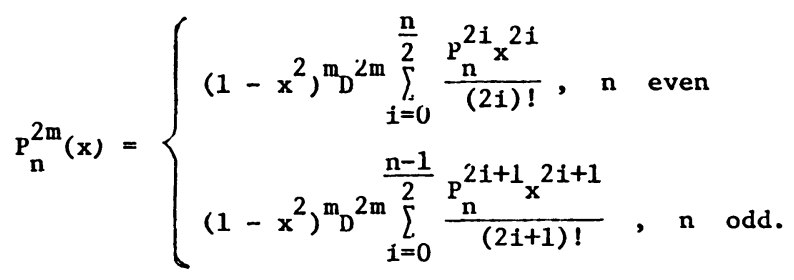

Taking the indicated derivatives in (2.4) gives

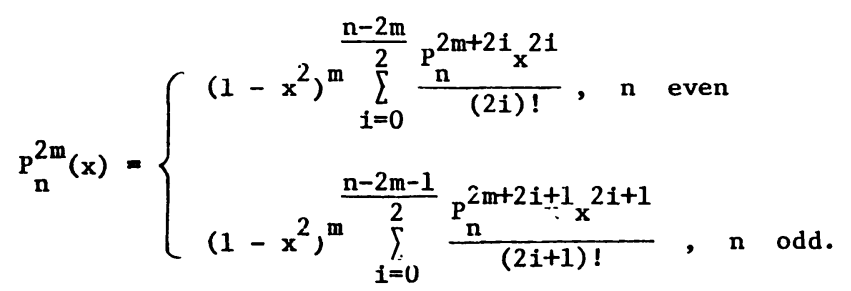

'lo obtain $D^{k} P_{n}^{2 m}(x)$, Leibniz' Theorem is used. For $0 \leq k \leq n$, one has from $(2.5)$

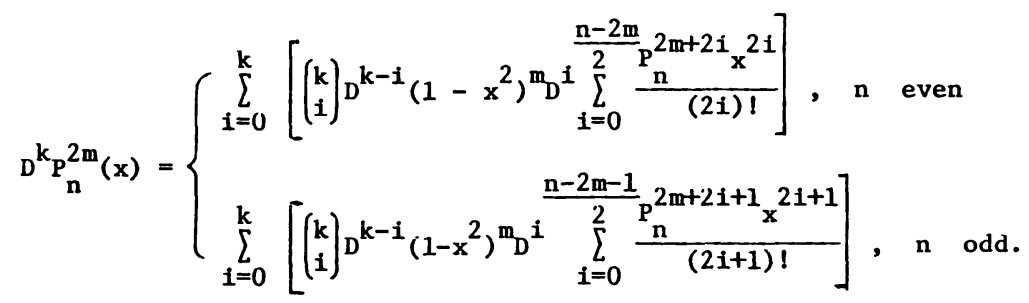

From this equation one sees that for $n$ even, $D^{k} P_{n}^{2 m}(x) j_{x=0}=0$ for $k$ odd, since in each term the first factor is 0 for $i$ even and the second is $U$ for $i$ odd. For $n$ odd, $\left.D^{k} P_{n}^{2 m}(x)\right]_{x=0}=0$ for $k$ even, since in each term the first factor is 0 for $i$ odd and the second is 0 for $i$ even.

One observes that for $n$ even,

$$
\left.D^{i} \sum_{i=0}^{\frac{n}{2}} \frac{P_{n}^{2 m+2 i} x^{2 i}}{(2 i) !}\right]_{x=0}=\left\{\begin{array}{l}
0, i \text { odd } \\
0, i>n-2 m \\
P_{n}^{2 m+i}, \quad i \text { even, } i \leq n-2 m,
\end{array}\right.
$$

and for $\mathbf{n}$ odd,

$$
\left.D^{i} \sum_{i=0}^{\frac{n-2 m-1}{2}} \frac{P_{n}^{2 m+2 i+1} x^{2 i+1}}{(2 i+1) !}\right]_{x=0}=\left\{\begin{array}{l}
0, i \text { even } \\
0, i>n-2 m \\
P_{n}^{2 m+i}, i \text { odd, } i \leq n-2 m
\end{array}\right.
$$

Further, 


$$
\left.D^{k-i}\left(1-x^{2}\right)^{m}\right]_{x=0}=\left\{\begin{array}{l}
0, k-i \text { odd } \\
0, k-i>2 m \\
\left.D^{k-i} \sum_{j=0}^{m}(-1)^{j}\left({ }_{j}^{m}\right) x^{2 j}\right]_{x=0}, k-i \text { even, } k-1 \leq 2 m .
\end{array}\right.
$$

The derivative on the right in (2.9) can be expressed using factorial notation as

$$
\left.\sum_{j=\frac{k-i}{2}}^{m}(-1)^{j}\left(\stackrel{m}{j}_{j}\right)(2 j)^{(k-i)} x^{2 j-k+i}\right]_{x=0}, k-i \text { even, } k-i \leq 2 m,
$$

which reduces to the first term

$$
(-1)^{\frac{k-i}{2}}\left[\begin{array}{c}
m \\
\frac{k-i}{2}
\end{array}\right)(k-i) !, k-i \text { even, } \frac{k-i}{2} \leq m,
$$

for $x=0$. Now (2.9) can be expressed as

$$
\left.D^{k-i}\left(1-x^{2}\right)^{m}\right]_{x=0}=\left\{\begin{array}{l}
0, k-i \text { odd } \\
0, k-i>2 m \\
\frac{k-1}{2}\left(\begin{array}{c}
m \\
\frac{k-i}{2}
\end{array}\right)(k-i) !, k-i \text { even, } \frac{k-i}{2} \leq m
\end{array}\right.
$$

Using Equations (2.7), (2.8), and (2.10) in (2.5) and the observations following $(2.6)$, one sees that

$$
\left.D^{k} p_{n}^{2 m}(x)\right]_{x=0}=\left\{\begin{array}{l}
0, n \text { even, } k \text { odd } \\
0, n \text { odd, } k \text { even } \\
\sum_{i=0}^{k} \frac{(-1)^{\frac{k-i}{2}} k ! m ! P_{n}^{2 m+i}}{i !\left(\frac{k-i}{2}\right) !\left(m-\frac{k-i}{2}\right) !}, n \text { and } k \text { of the same parity, }
\end{array}\right.
$$

where a term in the series above is 0 if $k-i$ is odd or if $m<\frac{k-1}{2}$. Also, recall that $P_{n}^{2 m+i}=0$ for $i>n-2 m$, for $i$ odd and $n$ even, and for $i$ even and $\mathbf{n}$ odd.

Equation (2.11) provides a formula for evaluating $\left.D^{k} P_{n}^{2 m}(x)\right]_{x=0}$. of course, the answers obtained by (2.11) agree with those obtained by other methods -- and can be easily verified for small integers $k, m$, and $n$.

3. ASSOCIATED LEGENDRE POLYNOMIALS AND $x^{n}$ AS SERIES OF LEGENDRE POLYNOMIALS.

It is known that an associated Legendre polynomial can be expressed as a series of Legendre polynomials. Equation (2.11) and a table of Legendre numbers, see Table 1 , can be used to provide a formula for the coefficients in the series. To outline the method, let

$$
P_{n}^{2 m}(x) \equiv \sum_{i=0}^{n} A_{i} P_{i}(x)
$$

Take $\mathbf{n}$ derivatives to obtain $\mathbf{n}$ other identities,

$$
D^{k} P_{n}^{2 m}(x) \equiv \sum_{i=0}^{n} A_{i} D^{i} P_{1}(x), k=1,2,3, \ldots, n .
$$

In these $n+1$ identities let $x=0$. Use (2.11) on the left sides of the resulting $n+1$ identities and recall that $\left.D^{k} p_{i}(x)\right]_{x=0}=P_{i}^{k}$, see [1]. The right sides can be 
simplified by using $P_{i}^{k}=0$ for $k>i$. The system of $n+1$ identities in $n+1$ unknowns can be solved for the $A_{i}$ 's and (3.1) gives the desired expansion. The values of the $A_{i}$ 's can be obtained in the order $A_{n}, A_{n-1}, A_{n-2}, \ldots, A_{0}$, by

$$
\left\{\begin{aligned}
A_{n} & =\frac{\left.D_{n}^{n} P_{n}^{2 m}(x)\right]}{P_{n}^{n}} \\
A_{n-1} & =\frac{\left.D^{n-i} P_{n}^{2 m}(x)\right]_{x=0}-\sum_{k=n-i+1}^{n} A_{k} P_{k}^{n-1}}{P_{n-i}^{n-i}}, 1 \leq i \leq n \\
& =\frac{\left.D^{n-i} P_{n}^{2 m}(x)\right]_{x=0}-\sum_{k=1}^{\left[\frac{1}{2}\right]} A_{n-i+2 k} P_{n-1+2 k}^{n-i}}{P_{n-i}^{n-i}}, 1 \leq i \leq n,
\end{aligned}\right.
$$

where $\left[\frac{i}{2}\right]=\frac{i}{2}$ for $i$ even and $\frac{i-1}{2}$ for $i$ odd.

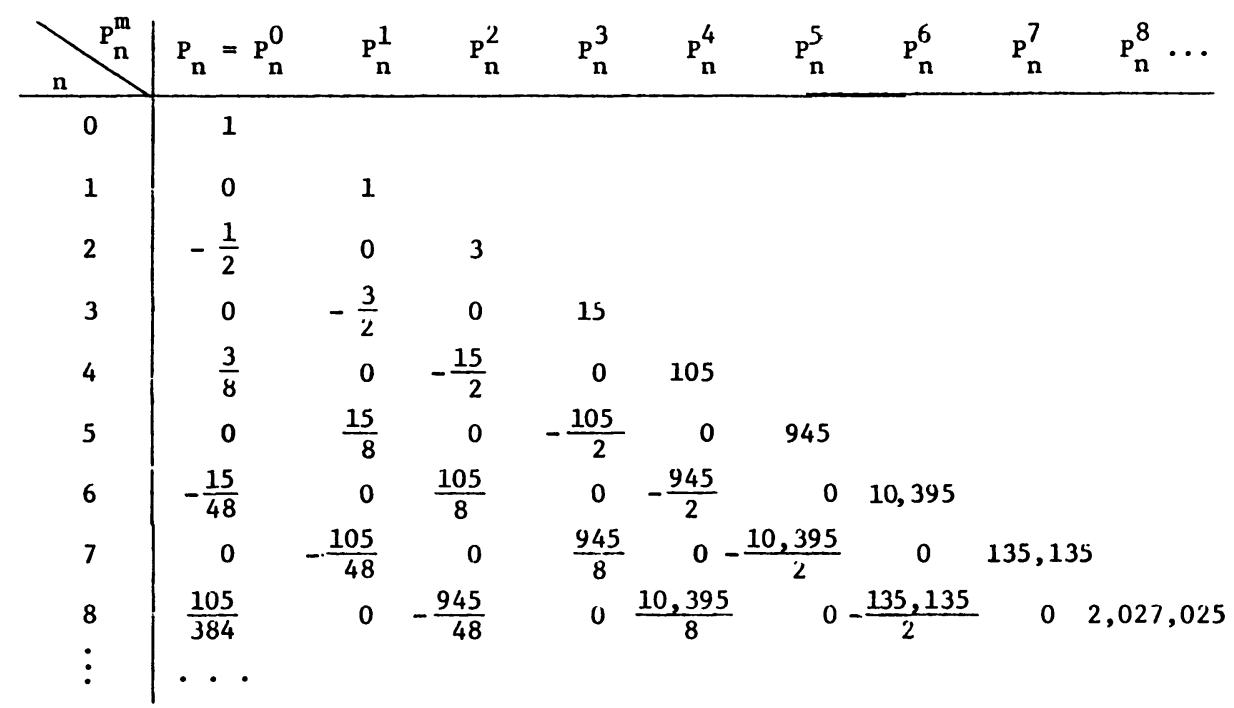

TABLE 1. LEGENDRE NUMBERS $\mathrm{P}_{\mathrm{n}}^{\mathrm{m}}$

Now, $x^{n}$ can be expanded in a series of Legendre polynomials in a similar way. Let

$$
x^{n} \equiv \sum_{i=0}^{n} A_{i} P_{i}(x)
$$

and proceed as in the derivation of (3.2) to obtain in order $A_{n}, A_{n-1}, A_{n-2}, \ldots, A_{0}$, as

$$
\begin{aligned}
& A_{n}=\frac{n !}{P_{n}^{n}}, \\
& A_{i}= \begin{cases}0, i=n-k, k \text { odd } \\
-\frac{1}{P_{i}^{i}} \quad \sum_{j=1}^{\frac{n-i}{2}} P_{i+2 j}^{i} A_{i+2 j}, \quad i=n-k, k \text { even. }\end{cases}
\end{aligned}
$$


With these values of the $A_{i}^{\prime}$ 's (3.3) gives the desired expansion, which agrees with the known expansion

$$
x^{n}=\frac{n !}{2^{n}} \sum_{k=0}^{\left[\frac{n}{2}\right]} \frac{(2 n-4 k+1) P_{n-2 k}(x)}{k !\left(\frac{3}{2}\right)_{n-k}} .
$$

4. SOME INTEGRALS INVOLVING LEGENDRE NUMBERS.

In [1], the result

$$
\int_{0}^{1} P_{n}(x) d x=-\frac{P_{n+1}}{n}
$$

for $n$ any positive integer is given. Here, two other important integrals are expressed in terms of Legendre numbers. It is known, see [2], that if $m=n$, then

$$
\int_{0}^{1} P_{n}(x) P_{m}(x) d x=\frac{1}{2 n+1}
$$

More generally, if $m$ and $n$ are different non-negative integers, Rainville, [2], gives the result

$$
\left.(n-m)(n+m+1) \int_{a}^{b} P_{n}(x) P_{m}(x) d x=\left(1-x^{2}\right)\left[P_{i n}^{\prime}(x) P_{n}(x)-P_{m}(x) P_{n}^{\prime}(x)\right]\right]_{a}^{b} \text {. }
$$

With $a=0, b=1$, and the results $\left.P_{n}^{m}=\frac{d^{m}\left(P_{n}(x)\right)}{d x}\right]_{x=0}, P_{1}^{n}=P_{m}^{1}=0$ for $m$ and $n$ even and $P_{n}=P_{m}=0$ for $m$ and $n$ odd from [1], Equation (4.3) becomes

$$
\begin{aligned}
\int_{0}^{1} P_{n}(x) P_{m}(x) & =\frac{P_{m}^{1} P_{n}-P_{m} P_{n}^{1}}{(m-n)(m+n+1)} \\
& =\left\{\begin{array}{l}
0, m \text { and } n \text { of the same parity } \\
\frac{P_{m}^{1} P_{n}}{(m-n)((m+n+1)}, m \text { and } n \text { of different parity. }
\end{array}\right.
\end{aligned}
$$

A third integral can be evaluated as shown by the following computation. With $\mathrm{n} \geq 1$

$$
\begin{aligned}
\int_{0}^{1} x_{n} P_{n-2 k}(x) d x & =\int_{0}^{1}\left[x_{m=0}^{n} \sum_{m !}^{n-2 k} \frac{P_{n-2 k}^{m}}{m !}\right] d x \\
& =\int_{0}^{1}\left(\sum_{m=0}^{n-2 k} \frac{P_{n-2 k}^{m} x^{m+n}}{m !}\right) d x \\
& =\sum_{m=0}^{n-2 k} \int_{0}^{1} \frac{P_{n-2 k}^{m}}{m !} d x \\
& \left.=\sum_{m=0}^{n-2 k} \frac{P_{n-2 k}^{m} x^{m+n+1}}{m !(m+n+1)}\right]_{0}^{1} \\
& =\sum_{m=0}^{n-2 k} \frac{P_{n-2 k}^{m}}{m !(m+n+1)} \cdot
\end{aligned}
$$

Therefore,

$$
\int_{0}^{1} x^{n} P_{n-2 k}(x) d x=\sum_{m=0}^{n-2 k} \frac{P_{n-2 k}^{m}}{m !(m+n+1)} .
$$

Since the value of this integral is known one can use this value for the series. 
Thus,

$$
\sum_{m=0}^{n-2 k} \frac{P_{n-2 k}^{m}}{m !(m+n+1)}=\frac{n !}{2^{n} \cdot k !\left(\frac{3}{2}\right)_{n-k}} .
$$

5. POLYNOMIALS AS LINEAR COMBINATIONS OF LEGENDRE POLYNOMIALS.

Since the Legendre polynomials form a simple set, any polynomial of a single variable can be expressed as a finite series of Legendre polynomials. Using Table 2, this can be done in much the same way any polynomial can be expressed in terms of factorials. Consider the problem of expressing

$$
H(x)=5 x^{3}-3 x^{2}+4 x-3
$$

in terms of Legendre polynomials. By continued subtraction of Legendre polynomials we obtain a zero remainder. Detaching coefficients,

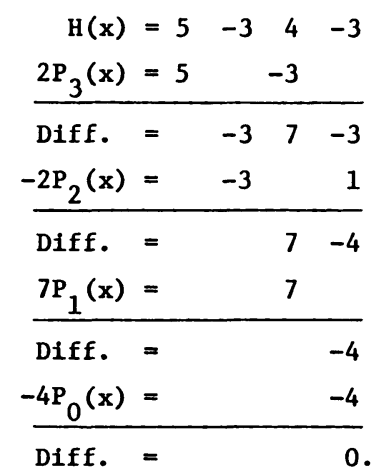

Thus

from which

$$
H(x)-2 P_{3}(x)+2 P_{2}(x)-7 P_{1}(x)+4 P_{0}(x)=0,
$$

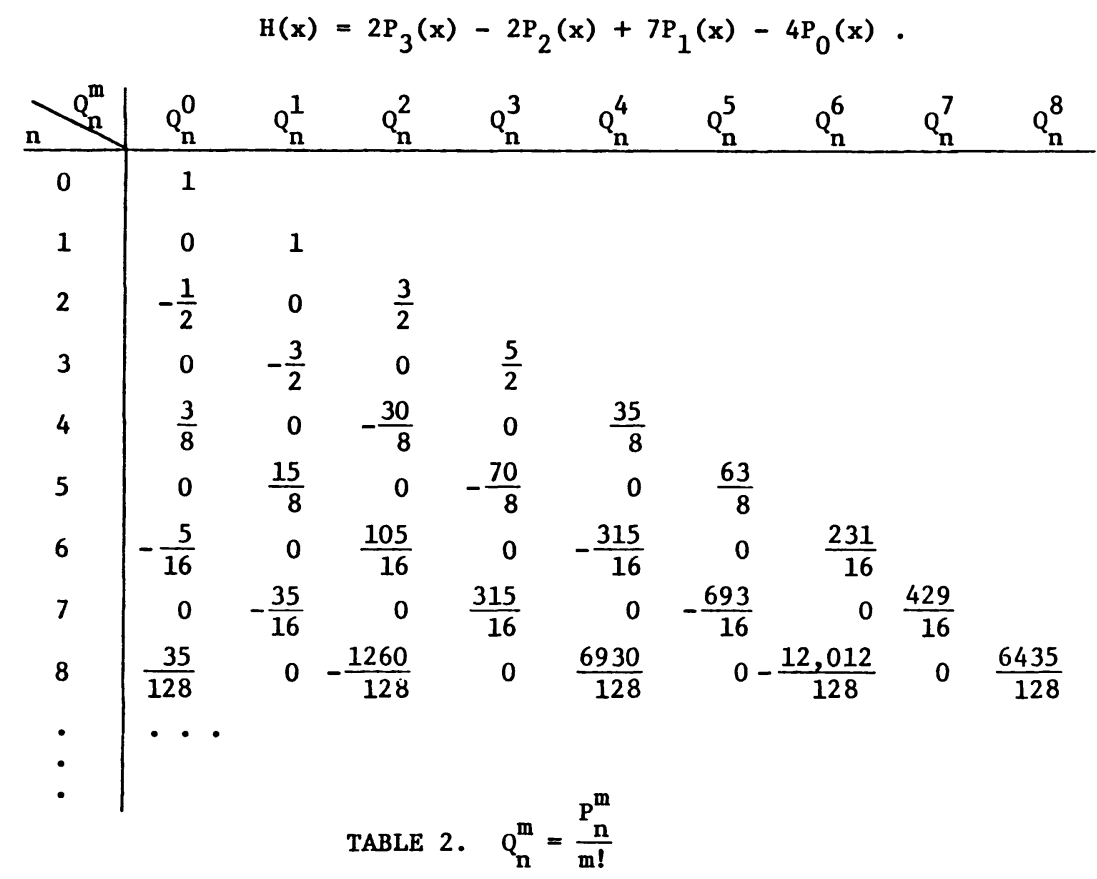


For a second method, set

$$
5 x^{3}-3 x^{2}+4 x-3 \equiv \mathrm{AP}_{3}(x)+\mathrm{BP}_{2}(\mathrm{x})+\mathrm{CP}_{1}(\mathrm{x})+\mathrm{DR}_{0}(\mathrm{x}) \text {. }
$$

Take the first three derivatives with respect to $x$, let $x=0$ in each of the four identities, and use $P_{n}^{m}(0)=P_{n}^{m}$ to obtain the system

$$
\left\{\begin{aligned}
-3 & =\mathrm{AP}_{3}+\mathrm{BP}_{2}+\mathrm{CP}_{1}+\mathrm{DP}_{0} \\
4 & =\mathrm{AP}_{3}^{1}+\mathrm{BP}_{2}^{1}+\mathrm{CP}_{1}^{1} \\
-6 & =\mathrm{AP}_{3}^{2}+\mathrm{BP}_{2}^{2} \\
30 & =\mathrm{AP}_{3}^{3}
\end{aligned}\right.
$$

Next, use Table 1 and solve for $A=2, B=-2, C=7$, and $D=-4$ in the order listed to obtain (5.1) again.

More generally, if $v(x)$ is a polynomial of degree $n$ in $x$, write

$$
v(x)=\sum_{i=0}^{n} A_{i} P_{i}(x),
$$

take $n$ derivatives, let $x=0$ in the $n+1$ identities, and use $P_{n}^{(m)}(0)=P_{n}^{m}$ to obtain the $A_{i}$ 's in the order $i=n$ to $i=0$ as

$$
\left\{\begin{array}{l}
A_{n}=\frac{v^{(n)}(0)}{P_{n}^{n}} \\
A_{i}=\frac{v^{(i)}(0)-\sum_{j=n-i+1}^{n} A_{j} P_{j}^{n-i}}{P_{n-i}^{n-i}}, 1=n-1, n-2, \ldots, 1,0
\end{array}\right.
$$

Since $P_{n}^{m}=0$ for $m+n$ odd, the second equation of (5.4) can be expressed as

$$
A_{i}=\frac{v^{(i)}(0)-\sum_{j=1}^{\frac{n-i}{2}} A_{i+2 j} P_{i+2 j}}{P_{i}^{i}}, i=n-1, n-2, .,, 1,0
$$

Table 2 can be used to evaluate a finite series of Legendre polynomials as a polynomial in $x$. As an example, we evaluate

Detaching coefficients,

$$
S(x)=P_{7}(x)-4 P_{6}(x)-5 P_{5}(x) \text {. }
$$

$$
\begin{array}{rrrrrrrrr}
P_{7}(x) & =0 & -\frac{35}{16} & 0 & \frac{315}{16} & 0 & -\frac{693}{16} & 0 & \frac{429}{16} \\
-4 P_{6}(x) & =\frac{5}{4} & 0 & -\frac{105}{4} & 0 & \frac{315}{4} & 0 & -\frac{231}{4} & \\
-5 P_{5}(x) & =0 & -\frac{75}{8} & 0 & \frac{350}{8} & 0 & -\frac{315}{8} & & \\
\hline \text { Sum } & =\frac{5}{4} & -\frac{185}{16} & -\frac{105}{4} & \frac{1015}{16} & \frac{315}{4} & -\frac{1323}{16} & -\frac{231}{4} & \frac{429}{16}
\end{array}
$$

Then,

$$
S(x)=\frac{5}{4}-\frac{185}{16} x-\frac{105}{4} x^{2}+\frac{1015}{16} x^{3}+\frac{315}{4} x^{4}-\frac{1323}{16} x^{5}-\frac{213}{4} x^{6}+\frac{429}{16} x^{7}
$$

6. LEGENDRE AND PASCAL NUMBERS.

Consider Table 3, which gives values for $L_{n^{m}}^{m}$ The entries shown are integers, a result that can be easily proved. The alternate diagonals have entries of the form 


$$
\frac{2^{n+1} P_{n+i}^{n-i}}{(n-i) !}, i=0 \text { to } n
$$

reading from upper right to lower left. If $2^{n} P_{n}^{n} / n$ ! is factored from

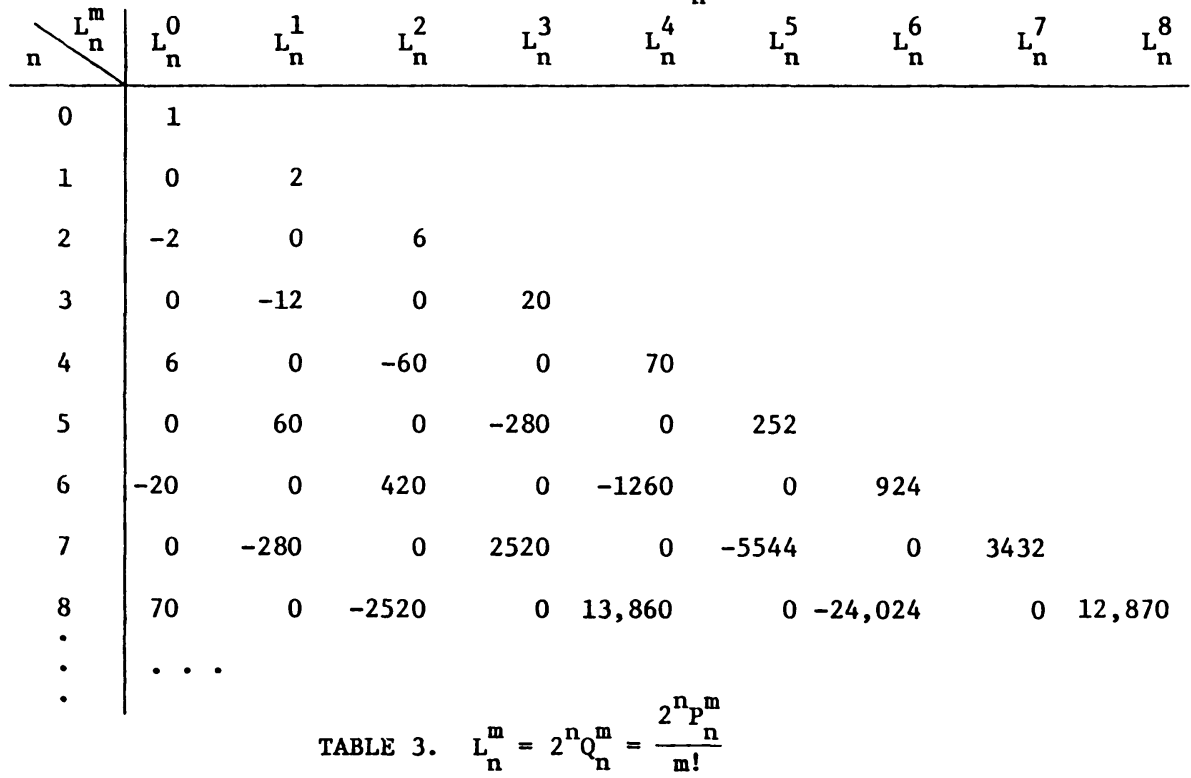

each entry on such a diagonal, the remaining factors are $(-1)^{1} c(n, 1)$. In notation, one has

$$
\frac{2^{n+1} P_{n+1}^{n-i}}{(n-i) !}=\frac{(-1)^{i} 2^{n} P_{n}^{n} C(n, i)}{n !}, i=0 \text { to } n \text {. }
$$

Equation (6.2) can be simplified to

$$
\frac{(-1)^{i} n ! 2^{i} P_{n+i}^{n-i}}{(n-1) ! P_{n}^{n}}=c(n, i), i=0 \text { to } n \text {, }
$$

which shows a connection between Legendre numbers and Pascal numbers. This result can be easily proved using the general form of the Legendre numbers, $P_{n}^{m}$, given in [1].

\section{REFERENCES}

1. HAGGARD, P.W. On Legendre Numbers, International Journal of Mathematics and Mathematical Sciences, Volume 8, Number 2, 1985, 407-411.

2. RAINVILLE, E.D. Special Functions, The Macmillan Company, New York, 1960.

3. COPSON, E.T. An Introduction to the Theory of Functions of a Complex Variable, Oxford University Press, London, 1935.

4. RICHARDSON, C.H. An Introduction to the Calculus of Finite Differences, C. Van Nostrand Company, Inc., New York, 1954. 


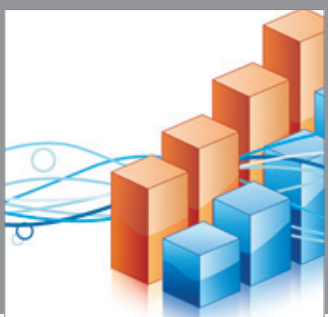

Advances in

Operations Research

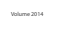



\section{The Scientific} World Journal
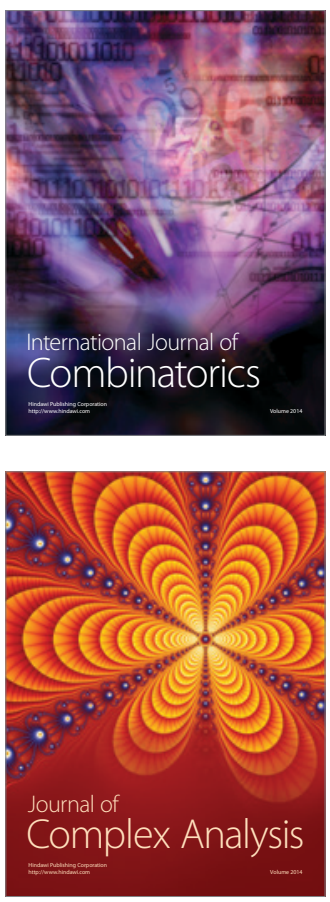

International Journal of

Mathematics and

Mathematical

Sciences
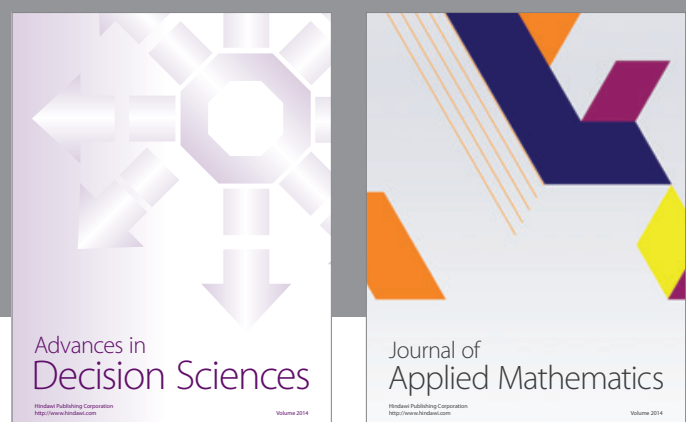

Journal of

Applied Mathematics
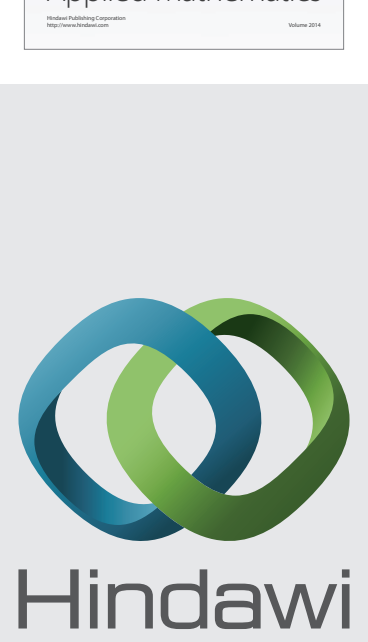

Submit your manuscripts at http://www.hindawi.com
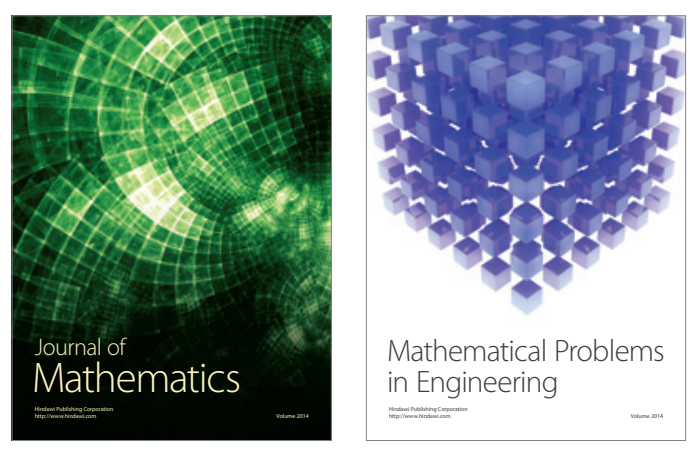

Mathematical Problems in Engineering
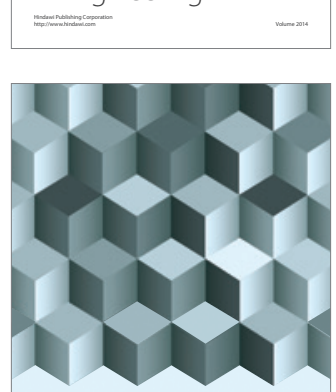

Journal of

Function Spaces
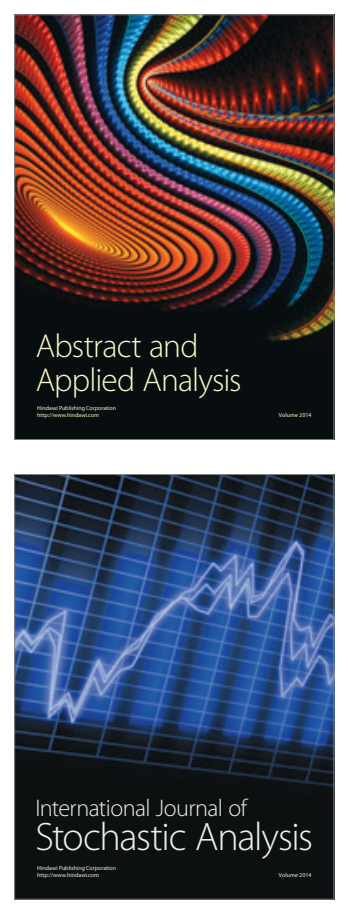

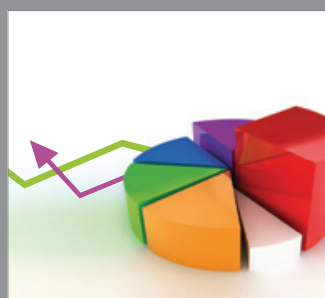

ournal of

Probability and Statistics

Promensencen
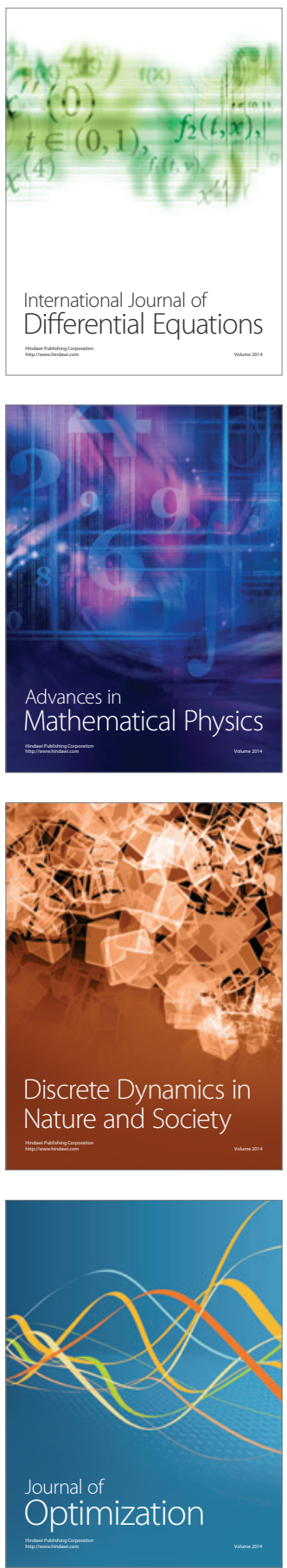